\title{
Alpha-Tocopherol Reduces Brain Edema and Protects Blood-Brain Barrier Integrity following Focal Cerebral Ischemia in Rats
}

\author{
Adel Haghnejad Azar ${ }^{\mathrm{a}}$ Shahrbanoo Oryan ${ }^{\mathrm{a}}$ Shahab Bohlooli ${ }^{\mathrm{b}}$ \\ Hamdollah Panahpour ${ }^{c}$ \\ ${ }^{a}$ Faculty of Biological Sciences, Kharazmi University, Tehran, and ${ }^{b}$ Department of Pharmacology, Pharmacy School, \\ and ${ }^{\mathrm{C} D e p a r t m e n t}$ of Physiology, Medical School, Ardabil University of Medical Sciences, Ardabil, Iran
}

\section{Key Words}

a-Tocopherol · Cerebral ischemia · Brain edema ·

Malondialdehyde · Glutathione

\begin{abstract}
Objective: This study was conducted to examine the neuroprotective effects of a-tocopherol against edema formation and disruption of the blood-brain barrier (BBB) following transient focal cerebral ischemia in rats. Materials and Methods: Ninety-six male Sprague-Dawley rats were divided into 3 major groups ( $n=32$ in each), namely the sham, and control and a-tocopherol-treated (30 mg/kg) ischemic groups. Transient focal cerebral ischemia ( $90 \mathrm{~min}$ ) was induced by occlusion of the left middle cerebral artery. At the end of the 24hour reperfusion period, the animals were randomly selected and used for 4 investigations $(n=8)$ in each of the 3 main groups: (a) assessment of neurological score and measurement of infarct size, (b) detection of brain edema formation by the wet/dry method, (c) evaluation of BBB permeability using the Evans blue (EB) extravasation technique, and (d) assessment of the malondialdehyde (MDA) and reduced glutathione (GSH) concentrations using high-performance liquid chromatography methods. Results: Induction of cerebral ischemia in the control group produced extensive brain edema (brain water content $83.8 \pm 0.11 \%$ ) and EB leakage into
\end{abstract}

\begin{tabular}{ll}
\hline KARGER & ( 2016 S. Karger AG, Basel \\
$\begin{array}{l}\text { E-Mail karger@karger.com } \\
\text { www.karger.com/mpp }\end{array}$ & $\begin{array}{l}\text { This is an Open Access article licensed under the terms of the } \\
\text { Creative Commons Attribution-NonCommercial 3.0 Un- } \\
\text { ported license (CC BY-NC) (www.karger.com/OA-license), } \\
\text { applicable to the online version of the article only. Distribu- } \\
\text { tion permitted for non-commercial purposes only. }\end{array}$
\end{tabular}

brain parenchyma $(14.58 \pm 1.29 \mu \mathrm{g} / \mathrm{g})$ in conjunction with reduced GSH and elevated MDA levels (5.86 $\pm 0.31 \mathrm{mmol} / \mathrm{mg}$ and $63.57 \pm 5.42 \mathrm{nmol} / \mathrm{mg}$, respectively). Treatment with a-tocopherol significantly lowered brain edema formation and reduced EB leakage compared with the control group ( $p<0.001,80.1 \pm 0.32 \%$ and $6.66 \pm 0.87 \mu \mathrm{g} / \mathrm{g}$, respectively). Meanwhile, treatment with a-tocopherol retained tissue GSH levels and led to a lower MDA level ( $p<0.01,10.17 \pm$ $0.83 \mathrm{mmol} / \mathrm{mg}$, and $\mathrm{p}<0.001,26.84 \pm 4.79 \mathrm{nmol} / \mathrm{mg}$, respectively). Conclusion: Treatment with a-tocopherol reduced ischemic edema formation and produced protective effects on BBB function following ischemic stroke occurrence. This effect could be through increasing antioxidant activity.

(c) 2016 S. Karger AG, Basel

\section{Introduction}

Brain edema is an abnormal accumulation of fluid in the brain parenchyma, and is a dangerous complication of stroke as it worsens the primary ischemic injury of the brain [1]. Prevention of brain edema formation may reduce mortality rates in ischemic stroke. Ischemic brain edema happens in 2 phases: cytotoxic edema, which develops within the first minutes to hours following ischemia, and vasogenic edema, which is permanent and takes 
hours to days to occur [2]. Vasogenic edema is characterized by an enlarged extracellular volume due to damage to the brain capillaries and leakage of plasma proteins such as albumin [3]. Vasogenic edema increases the risk for hemorrhage from impaired vessels [4] and cerebral herniation [2].

Oxidative stress has been shown to be involved in ischemia/reperfusion neuronal injury [5]. Reactive oxygen species (ROS) generated during cerebral ischemia and reduction of the brain antioxidant capacity are the most important causes of ischemic neuronal damage [6]. Incremental ROS production causes damage to lipid membranes and nucleic acids, and may lead to cell death. Generation of the ROS is further increased by reperfusion because an abrupt return of glucose and oxygen to the neurons causes free radical production at higher levels [7]. Strategies based on antioxidant supplementation have been shown to produce protective effects against brain injury in experimental models [8]. $\alpha$-Tocopherol, as a potent antioxidant, is the most important form of vitamin E. It has been reported that exogenous administration of $a$-tocopherol produces neuroprotective effects against cerebral ischemic injury, most probably by a reduction of ROS formation and the inhibition of inflammation and apoptosis $[9,10]$. However, there are no data about the effects of $\alpha$-tocopherol on vasogenic brain edema formation and blood-brain barrier (BBB) disruption following ischemia. Therefore, this study was conducted to investigate the effects of $\alpha$-tocopherol supplementation on ischemic brain edema formation, $\mathrm{BBB}$ permeability and oxidative stress biomarkers in the rat brain following transient focal cerebral ischemia.

\section{Materials and Methods}

\section{Animals}

Ninety-six male normotensive Sprague Dawley rats (280-320 g) were obtained from the central animal house facility of Ardabil Medical Sciences University, Ardabil, Iran. All protocols of the study were approved by the Institutional Animal Ethics Committee of Ardabil Medical Sciences University, which follows the NIH guidelines for the care and use of animals. The animals were food fasted overnight but had access to water. Anesthesia was performed intraperitoneally by injecting chloral hydrate $(400 \mathrm{mg} / \mathrm{kg})$. Body temperatures were maintained at $37 \pm 0.5^{\circ} \mathrm{C}$ with a heating feedback control system.

\section{Induction of Transient Focal Cerebral Ischemia}

Ninety-minute middle cerebral artery occlusion (MCAO) and 24-hour reperfusion of the left cerebral hemisphere were induced by the intraluminal filament method $[11,12]$. Regional cerebral blood flow (rCBF) was recorded from the cerebral cortex of the left hemisphere in the supply region of the middle cerebral artery (MCA) by means of a laser Doppler flowmeter pencil probe (MNP100; AD Instruments, Bella Vista, Australia). Baseline rCBF values measured before MCAO were defined as $100 \%$. MCAO was accepted as a decrease in $\mathrm{rCBF}$ to lower than $20 \%$ of the baseline value.

\section{Experimental Protocol}

Experiments were performed on 3 main groups of randomly divided animals:

Group 1 (sham, $\mathrm{n}=32$ ), rats underwent surgery at the neck region without being exposed to $\mathrm{MCAO}$ and received the vehicle $(1 \mathrm{ml} / \mathrm{kg})$ and $1 \%$ dimethyl sulfoxide intraperitoneally at the end of surgery.

Group 2 (control ischemic; $\mathrm{n}=32$ ), ischemia was induced by $90 \mathrm{~min}$ of MCAO followed by $24 \mathrm{~h}$ of reperfusion and the animals were treated with vehicle at the beginning of the reperfusion time.

Group 3 ( $\alpha$-tocopherol-treated ischemic rats; $n=32$ ), ischemia was induced and reperfusion performed as per group 2, after which a-tocopherol (30 mg/kg; LKT laboratories, St. Paul, MN, USA) was given at the beginning of the reperfusion time, as described previously [8].

Four sets of animals $(n=8)$ were studied in each main group. The first set was used for evaluation of the neurological score and measurement of infarct size. The second set of animals was selected to determine brain edema formation. Detection of BBB permeability was the objective for the third set of rats. The fourth set was used for assessment of the oxidative stress biomarkers (glutathione, GSH, and malondialdehyde, MDA). Rats that had incomplete MCAO and did not show $\mathrm{rCBF}<20 \%$ of baseline $(\mathrm{n}=1$ in group 3 ) or died within $24 \mathrm{~h}$ after ischemia ( $\mathrm{n}=1$ in group 2$)$ were excluded from the study $(\mathrm{n}=2)$.

\section{Assessment of Neurological Outcome}

A blinded observer assessed the neurological deficit $24 \mathrm{~h}$ after surgery. A 5-point scale of the neurological deficit score (NDS) test was carried out as described by Panahpour and Dehghani [13]. Briefly, the rats were graded as follows: normal motor function, 1; flexion of the contralateral torso or forelimb upon lifting of the tail, 2 ; circling to the contralateral side of the occlusion, 3; loss of righting reflex and decreased resistance to lateral push, 4 , and no spontaneous motor activity, 5.

\section{Measurement of the Cerebral Infarct Size}

Sections of the brain were cut into six 2 -mm-thick slices in the coronal plane with a brain matrix. The slices were then stained with a $2 \%$ solution of 2,3,5-triphenyltetrazolium chloride (TTC; Molekula, Newcastle upon Tyne, UK) at $37^{\circ} \mathrm{C}$ for $15 \mathrm{~min}$ and fixed in buffered formalin. Images of the stained sections were obtained and the infarction areas were quantified using Scion image analyzer software [13].

\section{Detection of Brain Edema Formation}

Ischemic brain edema was assessed using the dry/wet method [14]. Each hemisphere of the brain was placed in a distinct preweighed dish to measure its wet weight (WW). Then the dish and the tissues were placed in a $110^{\circ} \mathrm{C}$ oven for $24 \mathrm{~h}$ to find the tissue dry weight (DW). The absolute brain water content (WC) of each hemisphere (\%) was calculated using the following equation:

$\mathrm{WC}(\%)=[(\mathrm{WW}-\mathrm{DW}) / \mathrm{WW}] \times 100$.
18

Med Princ Pract 2017;26:17-22

DOI: $10.1159 / 000450648$
Haghnejad Azar/Oryan/Bohlooli/ Panahpour 
Fig. 1. $\mathrm{rCBF}$ in control ischemic rats received vehicle $(\mathrm{n}=8)$ and ischemic rats received a-tocopherol at a dose of $30 \mathrm{mg} / \mathrm{kg}$ $(\mathrm{n}=8)$ before MCAO, during MCAO and during reperfusion times.

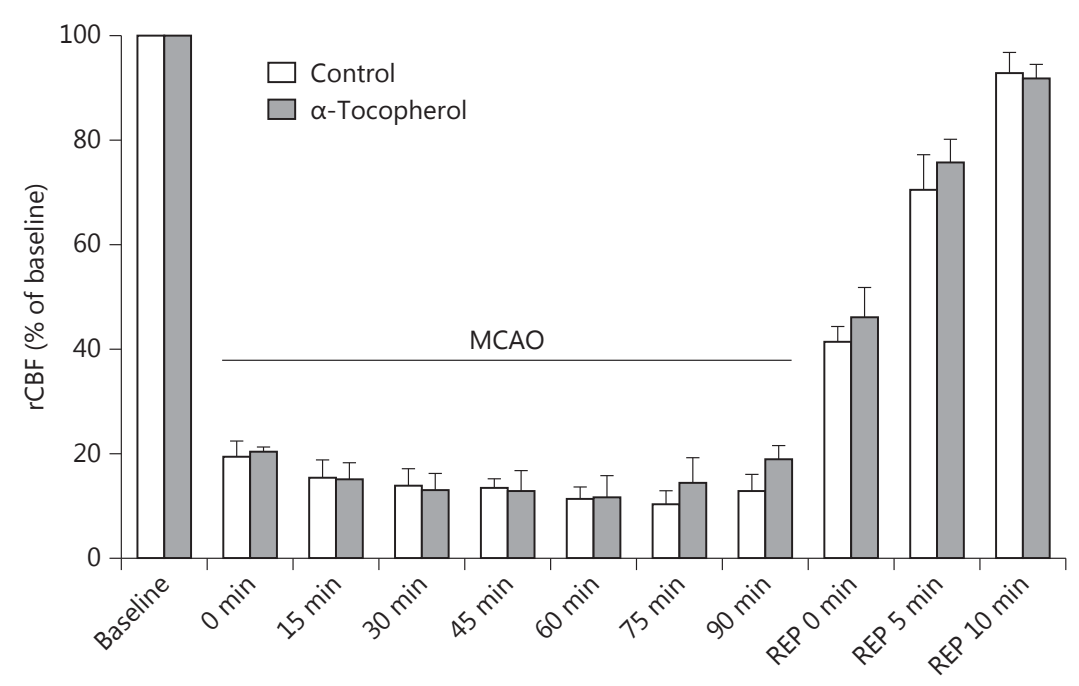

\section{Assessment of BBB Permeability}

BBB permeability was estimated with the Evans blue (EB) extravasation technique [15]. The EB infusion (i.v. $1 \mathrm{ml} / \mathrm{kg}$ of $2 \% \mathrm{~EB}$ solution in saline) was started $30 \mathrm{~min}$ after surgery and continued for $5 \mathrm{~min}$. After $24 \mathrm{~h}$ the chest was opened under deep anesthesia. The whole body was then perfused with about $250 \mathrm{ml}$ of warm saline solution $\left(37^{\circ} \mathrm{C}\right)$ infused into the left ventricle to wash the EB dye out of the circulation.

After decapitation of the animal, the brain was divided into right and left hemispheres. Each hemisphere was weighed and homogenized in $2.5 \mathrm{ml}$ of phosphate-buffered saline. The homogenized solution was mixed with $2.5 \mathrm{ml}$ of trichloroacetic acid (60\%) and centrifuged for $30 \mathrm{~min}$ at 3,500 rpm $(1,640 \mathrm{~g})$. The collected supernatant was used to determine the EB absorbance at $610 \mathrm{~nm}$ by a spectrophotometer (UV 1800; Shimadzu, Kyoto, Japan). EB concentrations were calculated against a standard curve and the obtained results are expressed as micrograms per gram of brain tissue.

\section{Assessment of Oxidative Stress Biomarkers}

The animals were reperfused for $24 \mathrm{~h}$, after which they were decapitated and the brain was removed. The ischemic area of the lesion in the hemisphere was separated according to well-established protocols in rodent models $[16,17]$. The tissue was weighed and homogenized in PBS with a weight-to-volume ratio of 1:5. The homogenate was centrifuged $\left(10,000 \mathrm{~g}, 4^{\circ} \mathrm{C}\right)$ for $30 \mathrm{~min}$. The supernatant was used for quantifying the oxidative stress biomarkers. Lipid peroxidation was evaluated by determining the concentration of MDA in the brain samples using the previously described high-performanceliquid chromatography (HPLC) method $[14,18,19]$. Results are expressed as tissue WW (nmol/mg). The concentration of reduced GSH as an important index of antioxidant defense capacity was also detected by the HPLC method [14, $18,20]$. Results are expressed as millimoles per milligram (mmol/ $\mathrm{mg}$ ) of wet tissue weight.

a-Tocopherol Reduces Ischemic Brain Edema

\section{Statistical Analyses}

The data are represented as the mean \pm SEM and the significance of differences were evaluated using one-way analysis of variance (ANOVA) followed by Tukey's test. Statistical significance was accepted at $\mathrm{p}<0.05$.

\section{Results}

\section{Recording of Cerebral Blood Flow and Physiological Parameters}

The baseline rCBF values recorded before MCAO were defined as $100 \%$. The rCBF values were reduced to less than $20 \%$ of baseline in both the control and treated ischemic groups after MCA occlusion. There was no significant difference between rCBF values in the groups during occlusion and the first $10 \mathrm{~min}$ of reperfusion (fig. 1). The physiological parameters measured were systolic blood pressure, heart rate, peripheral oxygen saturation $\left(\mathrm{SpO}_{2}\right)$, and body temperature before and after MCAO, which were within the normal physiological range, and inter- and intragroup comparisons indicated no significant differences among them.

\section{Evaluation of the NDS and Infarct Size}

Ischemia produced severe motor disabilities in control ischemic rats $(4 \pm 0.5)$. However, the NDS of ischemic rats treated with $\alpha$-tocopherol was $1.75 \pm 0.25$ and the difference was statistically significant compared to the control group ( $\mathrm{p}<0.05)$. Sham-operated rats had no infarctions. 


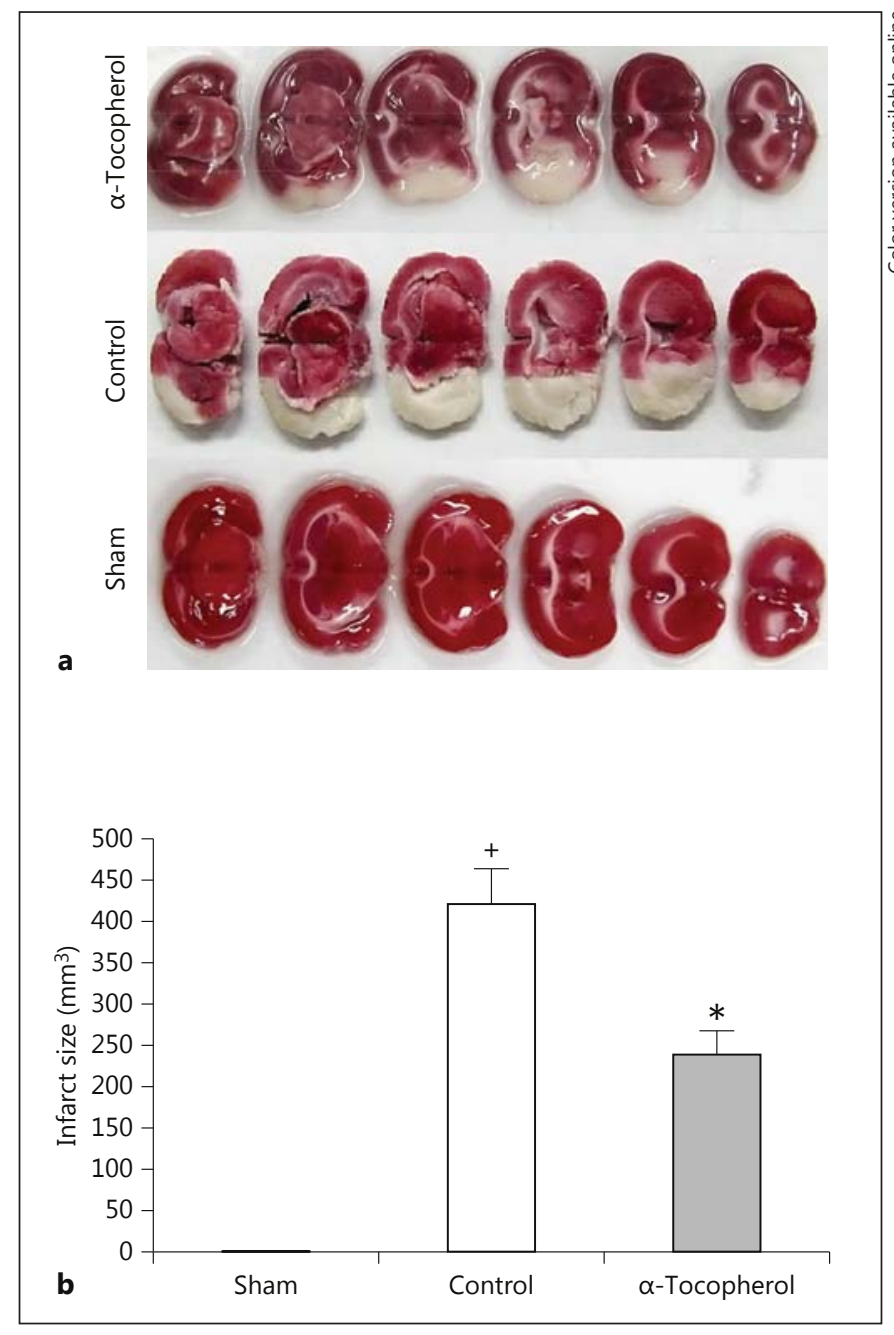

Fig. 2. a Representative brain slices stained with TTC in the studied groups. Ischemic regions are white and nonischemic regions are red (colors refer to the online version only). b Infarct size in the study groups. Values are means $\pm \operatorname{SEM}(n=8) .{ }^{+} \mathrm{p}<0.001$ versus sham group; $* \mathrm{p}<0.01$ versus control group.

MCA occlusion of the left side of the brain produced considerable cerebral lesions in the control-ischemia rats $\left(422 \pm 41 \mathrm{~mm}^{3}\right)$. Treatment with $\alpha$-tocopherol $(30 \mathrm{mg} / \mathrm{kg})$ significantly diminished cerebral lesion volumes by $42.9 \%$ $\left(241 \pm 29 \mathrm{~mm}^{3}, \mathrm{p}<0.01\right.$; fig. 2).

\section{Assessment of Ischemic Brain Edema}

There was no statistically significant difference between the water content of the right side of brains between the experimental groups $(79.1 \pm 0.28,79.2 \pm 0.34$ and $78.5 \pm 0.17 \%$ for the sham, control and $\alpha$-tocopheroltreated groups, respectively). Water content of the left

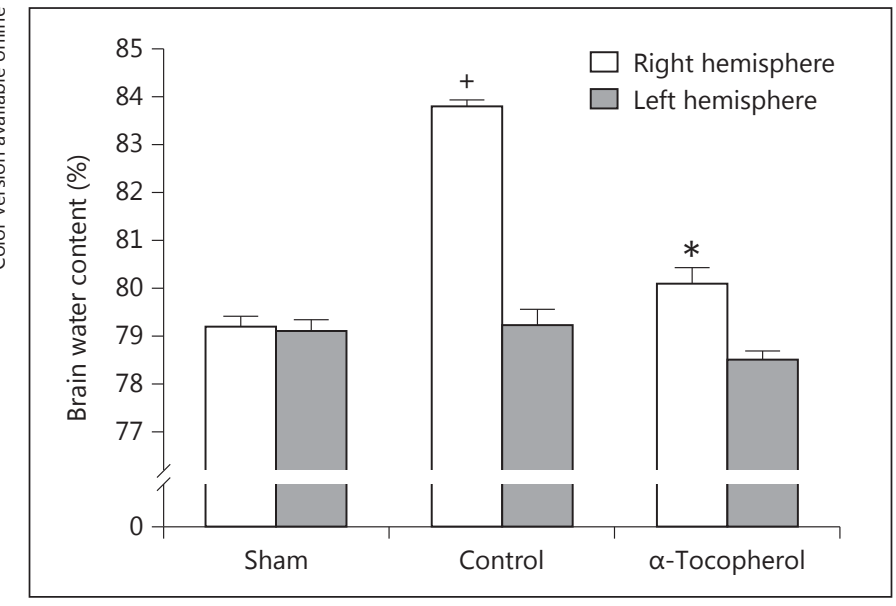

Fig. 3. Water content in the left and right sides of the brains in the study groups $(\mathrm{n}=8) .{ }^{+} \mathrm{p}<0.001$ compared with the sham group; $* \mathrm{p}<0.001$ compared with the control group.

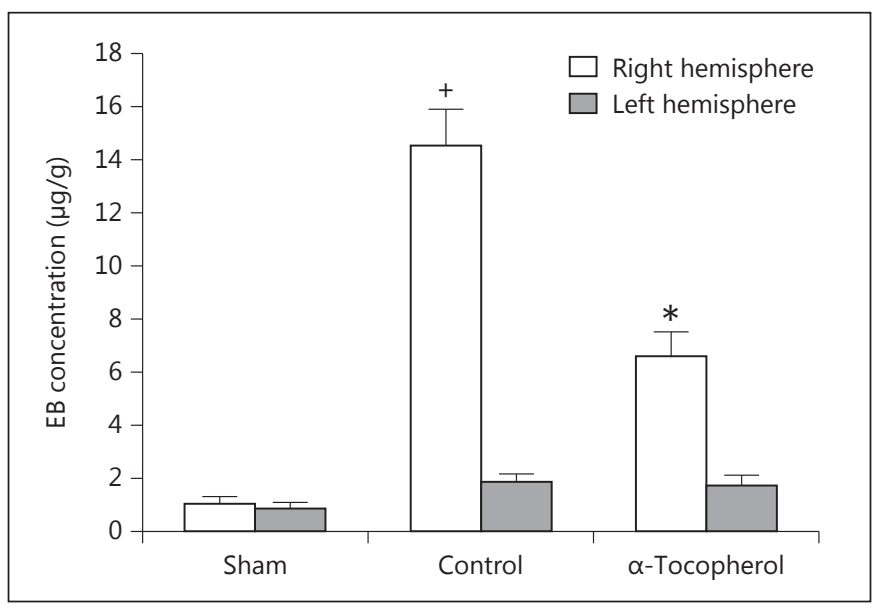

Fig. 4. EB concentration in the left and right sides of the brains in the study groups $(n=8) .{ }^{+} \mathrm{p}<0.001$ compared with the sham group; ${ }^{*} \mathrm{p}<0.001$ compared with the control group.

side of the brain in the control ischemic rats was significantly more than that of the sham-operated rats ( $\mathrm{p}<$ $0.001,83.8 \pm 0.11$ vs. $79 \pm 0.2 \%$, respectively). Postischemic treatment with $a$-tocopherol $(30 \mathrm{mg} / \mathrm{kg})$ was significantly associated with lower brain water content $(80.1 \pm 0.32 \%)$ than that of control ischemic rats $(\mathrm{p}<$ 0.001; fig. 3).

\section{Evaluation of BBB Permeability}

There was no statistically significant difference between the EB concentrations of the right side of brains between the groups $(0.87 \pm 0.22,1.88 \pm 0.29$ and $1.74 \pm$ 

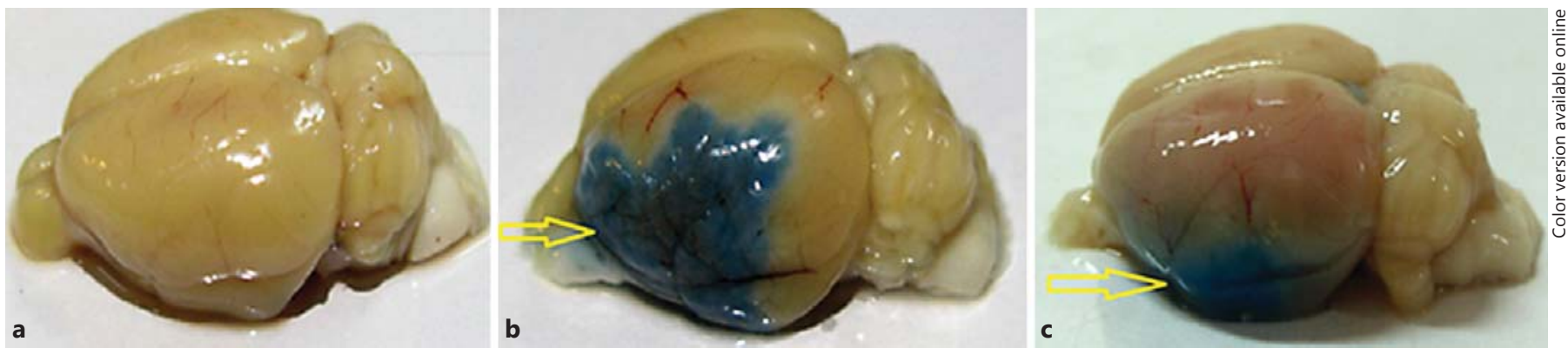

Fig. 5. Photographs of brains in the sham (a), control ischemic (b) and $\alpha$-tocopherol-treated $(30 \mathrm{mg} / \mathrm{kg} ; \mathbf{c})$ ischemic rats. The intensity of the blue color depicted in $\mathbf{b}$ and $\mathbf{c}$ (indicated by arrows; color refers to the online version only) is related to the extent of damage of the cerebral vasculature of the lesioned side.

$0.37 \mu \mathrm{g} / \mathrm{g}$ for the sham, control and $\alpha$-tocopherol-treated groups, respectively). For the left side of the brain, the EB concentration of control ischemic rats was significantly more than that of sham-operated rats $(\mathrm{p}<0.001,14.58 \pm$ 1.29 vs. $1.1 \pm .22 \mu \mathrm{g} / \mathrm{g}$, respectively). Postischemic treatment with a-tocopherol significantly lowered the EB concentration of the ischemic side of the brain $(\mathrm{p}<0.001$, $6.66 \pm 0.87 \mu \mathrm{g} / \mathrm{g}$; fig. 4, 5).

\section{Assessment of MDA and GSH}

The induction of ischemia and reperfusion in the control ischemic group resulted in significantly increased MDA concentrations $(63.57 \pm 5.42 \mathrm{nmol} / \mathrm{mg})$ and reduced GSH concentrations ( $5.86 \pm 0.31 \mathrm{mmol} / \mathrm{mg}$ ) in the left side of the brain tissues, as compared to the sham group ( $\mathrm{p}<0.001)$. Treatment with $\alpha$-tocopherol provided a significantly reduced MDA concentration ( $<<0.001$, $26.84 \pm 4.79 \mathrm{nmol} / \mathrm{mg}$ ) and elevated GSH concentration ( $\mathrm{p}<0.01,10.17 \pm 0.83 \mathrm{mmol} / \mathrm{mg}$ ) compared to the control ischemic group (table 1).

\section{Discussion}

This study showed that treatment with $\alpha$-tocopherol had protective effects against vasogenic edema formation based on reducing brain water content and EB leakage. This protective effect might be partly related to the inhibition of oxidative stress. As far as the literature is concerned, there is no report about the effects of a-tocopherol on edema formation and BBB destruction during ischemia. Stoffel et al. [21] reported that dietary supplementation of $\alpha$-tocopherol did not produce any significant influence against posttraumatic vasogenic brain edema. This dissimilarity in results might be re-

a-Tocopherol Reduces Ischemic Brain Edema
Table 1. Concentrations of reduced GSH and MDA in the study groups

\begin{tabular}{lll}
\hline Group & GSH, mmol/mg & MDA, nmol/mg \\
\hline Sham & $17.5 \pm 1.17$ & $17.12 \pm 1.48$ \\
Control & $5.86 \pm 0.31^{+}$ & $63.57 \pm 5.42^{+}$ \\
a-Tocopherol & $10.17 \pm 0.83^{*}$ & $26.84 \pm 4.79^{* *}$ \\
\hline
\end{tabular}

Values are the mean \pm SEM $(n=8) .{ }^{+} \mathrm{p}<0.001$ versus sham; ${ }^{*} \mathrm{p}<0.01,{ }^{* *} \mathrm{p}<0.001$ versus control.

lated to differences in pathophysiological mechanisms involved in traumatic or ischemic brain edema and the diverse methods used. However, our findings confirmed previous studies that had reported the neuroprotective effects for $\alpha$-tocopherol against ischemic brain damage $[9,10]$. It was demonstrated that vitamin E deficiency caused a greater neurological deficit and infarct volume [22] while exogenous administration of $\alpha$ - tocopherol reduced ischemic reperfusion-induced neuronal damage [9].

Oxidative stress caused by the excessive production of free radicals and overwhelming of brain antioxidant capacity has been shown to be involved in ischemic brain damage [23]. Compared to other tissues, the brain is more sensitive to oxidative damage because it has a high rate of metabolism, high lipid content and relative low protective antioxidant systems $[8,24]$. Our findings demonstrated that treatment with $\alpha$-tocopherol produced protective effects against oxidative damage of the brain. This protection was partly achieved by a reduction of lipid peroxidation and augmentation of brain antioxidant capacity via increased GSH. Previous studies showed that exogenous 
administration of $\alpha$-tocopherol attenuated raised levels of MDA after brain ischemia $[9,25]$. Additionally, it was reported that $\alpha$-tocopherol acts as an acyl-peroxyl-radical scavenger and inhibits superoxide anion production by leukocytes via reducing NADPH oxidase activity [26]. Thus, protective effects of $\alpha$-tocopherol against ischemic brain injury might be partly attributed to antioxidant actions. Beside the protective effects of $\alpha$-tocopherol against lipid peroxidation are its nonantioxidant properties, such as the preservation of endothelial integrity, inhibition of monocyte-endothelial adhesion, reduction of cytokine release and platelet aggregation [27-29]. In general, the inhibition of ROS formation, inflammation and apoptosis $[9,10]$ could be possible mechanisms. However, further studies are needed to clarify the exact mechanisms of beneficial $\alpha$-tocopherol effects.

\section{Conclusion}

In this study, postischemictreatment with $\alpha$-tocopherol attenuated vasogenic brain edema by protecting BBB integrity. In addition, the administration of $\alpha$-tocopherol amplified the brain antioxidant capacity. These data suggest that treatment with $\alpha$-tocopherol provided protective effects against ischemic edema formation, partly by antioxidant actions.

\section{Acknowledgement}

This work was supported by a grant from the Vice Chancellor for Research, Ardabil University of Medical Sciences, Ardabil, Iran. The authors would like to thank Nooshin Sadeghian for her technical assistance.

\section{References}

1 Barakat R, Redzic Z: The role of activated microglia and resident macrophages in the neurovascular unit during cerebral ischemia: is the jury still out? Med Princ Pract 2016;25:314.

2 Fishman RA: Cerebrospinal Fluid in Diseases of the Nervous System, ed 2. Philadelphia, Saunders, 1992, pp 103-155.

3 Marmarou A: The pathophysiology of brain edema and elevated intracranial pressure. Cleve Clin J Med 2004;71:S6-S8.

4 Rosenberg GA: Ischemic brain edema. Prog Cardiovasc Dis 1999;42:209-216.

5 Allen CL, Bayraktutan U: Oxidative stress and its role in the pathogenesis of ischaemic stroke. Int J Stroke 2009;4:461-470.

6 Miquel J: An update on the mitochondrialDNA mutation hypothesis of cell aging. Mutat Res 1992;275:209-216.

7 Chan PH: Role of oxidants in ischemic brain damage. Stroke 1996;27:1124-1129.

8 Margaill I, Plotkine M, Lerouet D: Antioxidant strategies in the treatment of stroke. Free Radic Biol Med 2005;39:429-443.

9 Chaudhary G, Sinha K, Gupta YK: Protective effect of exogenous administration of alphatocopherol in middle cerebral artery occlusion model of cerebral ischemia in rats. Fundam Clin Pharmacol 2003;17:703-707.

10 Hsiao G, Lee JJ, Chen YC, et al: Neuroprotective effects of PMC, a potent $\alpha$-tocopherol derivative, in brain ischemia-reperfusion: reduced neutrophil activation and anti-oxidant actions. Biochem Pharmacol 2007;73:682693.

11 Panahpour $\mathrm{H}$, Nouri M: Post-ischemic treatment with candesartan protects from cerebral ischemic/reperfusion injury in normotensive rats. Int J Pharm Pharm Sci 2012;4:286-289.

12 Panahpour H, Nekooeian AA, Dehghani GA: Candesartan attenuates ischemic brain edema and protects the blood-brain barrier integrity from ischemia/reperfusion injury in rats. Iran Biomed J 2014;18:232-238.

13 Panahpour H, Dehghani GA: Attenuation of focal cerebral ischemic injury following postischemic inhibition of angiotensin converting enzyme (ACE) activity in normotensive rat. Iran Biomed J 2012;16:202-208.

14 Panahpour H, Dehghani GA, Bohlooli S: Enalapril attenuates ischaemic brain oedema and protects the blood-brain barrier in rats via an anti-oxidant action. Clin Exp Pharmacol Physiol 2014;41:220-226.

15 Kaya M, Kucuk M, Kalayci RB, et al: Magnesium sulfate attenuates increased blood-brain barrier permeability during insulin-induced hypoglycemia in rats. Can J Physiol Pharmacol 2001;79:793-798.

16 Ashwal S, Tone B, Tian HR, et al: Core and penumbral nitric oxide synthase activity during cerebral ischemia and reperfusion. Stroke 1998;29:1037-1047.

17 Lie B, Popp S, Capuano-Waters C, et al: Lidocaine attenuates apoptosis in the ischemic penumbra and reduces infarct size after transient focal cerebral ischemia in rats. Neuroscience 2004; 125:691-701.

18 Nakhostin-Roohi B, Barmaki S, Khoshkhahesh F, et al: Effect of chronic supplementation with methylsulfonylmethane on oxidative stress following acute exercise in untrained healthy men. J Pharm Pharmacol 2011;63: 1290-1294.

19 Mateos R, Lecumberri E, Ramos S, et al: Determination of malondialdehyde by high-performance liquid chromatography in serum and liver as a biomarker for oxidative stress application to a rat model for hypercholesterolemia and evaluation of the effect of diets rich in phenolic antioxidant from fruits. J Chromatogr B 2005;827:76-82.
20 Giustarini D, Dalle-Donne I, Colombo R, et al: An improved HPLC measurement for GSH and GSSG in human blood. Free Radic Biol Med 2003;35:1365-1372.

21 Stoffel M, Berger S, Staub F, et al: The effect of dietary a-tocopherol on the experimental vasogenic brain edema. J Neurotrauma 1997;14: 339-348.

22 Stohrer M, Eichinger A, Schlachter M, et al: Protective effect of vitamin $\mathrm{E}$ in a rat model of focal cerebral ischemia. Z Naturforech C 1998; 53:273-278.

23 Wang Y, Chang CF, Chou J, et al: Dietary supplementation with blueberries, spinach, or spirulina reduces ischemic brain damage. Exp Neurol 2005;193:75-84.

24 Awad AS: Effect of combined treatment with curcumin and candesartan on ischemic brain damage in mice. J Stroke Cerebrovasc Dis 2011;20:541-548.

25 Tovmasyan A, Sheng H, Weitner T, et al: Design, mechanism of action, bioavailability and therapeutic effects of mn porphyrin-based redox modulators. Med Princ Pract 2013;22: 103-130.

26 Cachia O, Benna JE, Pedruzzi E, et al: Alphatocopherol inhibits the respiratory burst in human monocytes. Attenuation of p47(phox) membrane translocation and phosphorylation. J Biol Chem 1998;273:32801-32805.

27 Azzi A: Molecular mechanism of $\alpha$-tocopherol action. Free Radic Biol Med 2007;43:16-21.

28 van Aalst JA, Burmeister W, Fox PL, et al: Alpha-tocopherol preserves endothelial cell migration in the presence of celloxidized lowdensity lipoprotein by inhibiting changes in cell membrane fluidity. J Vasc Surg 2004;39: 229-237.

29 Devaraj S, Jialal I: The effects of $\alpha$-tocopherol on critical cells in atherogenesis. Curr Opin Lipidol 1998;9:11-15. 